VIII JORNADAS

SOBRE INNOVACIÓN DOCENTE

EN ARQUITECTURA

WORKSHOP ON EDUCATIONAL INNOVATION

IN ARCHITECTURE JIDA'20

JORNADES SOBRE INNOVACIÓ

DOCENT EN ARQUITECTURA JIDA'20

ESCUELA TÉCNICA SUPERIOR DE ARQUITECTURA DE MÁLAGA 12 Y 13 DE NOVIEMBRE DE 2020 


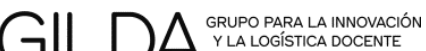 EN ARQUITECTURA}

Organiza e impulsa GILDA (Grupo para la Innovación y Logística Docente en la Arquitectura), en el marco del proyecto RIMA (Investigación e Innovación en Metodologías de Aprendizaje), de la Universitat Politècnica de Catalunya - BarcelonaTech (UPC) y el Institut de Ciències de l'Educació (ICE). http://revistes.upc.edu/ojs/index.php/JIDA

\section{Editores}

Berta Bardí i Milà, Daniel García-Escudero

\section{Revisión de textos}

Alba Arboix, Jordi Franquesa, Joan Moreno, Judit Taberna

\section{Edita}

Iniciativa Digital Politècnica Oficina de Publicacions Acadèmiques Digitals de la UPC Publicaciones y Divulgación Científica, Universidad de Málaga

ISBN 978-84-9880-858-2 (IDP-UPC) 978-84-1335-032-5 (UMA EDITORIAL)

eISSN 2462-571X

(C) de los textos y las imágenes: los autores

(C) de la presente edición: Iniciativa Digital Politècnica Oficina de Publicacions Acadèmiques Digitals de la UPC, UMA

Esta obra está sujeta a una licencia Creative Commons:

Reconocimiento - No comercial - SinObraDerivada (cc-by-nc-nd):

http://creativecommons.org/licences/by-nc-nd/3.0/es

https://creativecommons.org/licenses/by-nc-nd/4.0/

Cualquier parte de esta obra se puede reproducir sin autorización pero con el reconocimiento y atribución de los autores.

No se puede hacer uso comercial de la obra y no se puede alterar, transformar o hacer obras derivadas. 


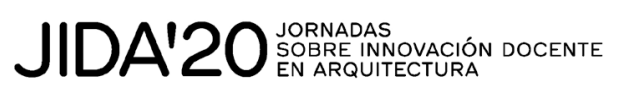

\section{Comité Organizador JIDA'20}

\section{Dirección y edición}

\section{Berta Bardí i Milà (GILDA)}

Dra. Arquitecta, Departamento de Proyectos Arquitectónicos, ETSAB-UPC

Daniel García-Escudero (GILDA)

Dr. Arquitecto, Departamento de Proyectos Arquitectónicos, ETSAB-UPC

\section{Organización}

\section{Antonio Álvarez Gil}

Dr. Arquitecto, Departamento Arte y Arquitectura, eAM'-UMA

\section{Jordi Franquesa (Coordinador GILDA)}

Dr. Arquitecto, Departamento de Urbanismo y Ordenación del Territorio, ETSAB-UPC Joan Moreno Sanz (GILDA)

Dr. Arquitecto, Departamento de Urbanismo y Ordenación del Territorio, ETSAV-UPC

\section{Fernando Pérez del Pulgar Mancebo}

Dr. Arquitecto, Departamento Arte y Arquitectura, eAM'-UMA

Judit Taberna (GILDA)

Arquitecta, Departamento de Representación Arquitectónica, ETSAB-UPC

Ferran Ventura Blanch

Dr. Arquitecto, Departamento Arte y Arquitectura, eAM'-UMA

\section{Coordinación}

\section{Alba Arboix}

Dra. Arquitecta, Teoría e Historia de la Arquitectura y Técnicas de la Comunicación, ETSAB-UPC

\section{Comunicación}

Eduard Llorens i Pomés

ETSAB-UPC 


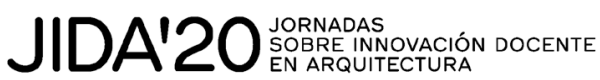

\section{Comité Científico JIDA'20}

Luisa Alarcón González

Dra. Arquitecta, Proyectos Arquitectónicos, ETSA-US

Gaizka Altuna Charterina

Arquitecto, Representación Arquitectónica y Diseño, TU Berlin

Atxu Amann Alcocer

Dra. Arquitecta, Ideación Gráfica Arquitectónica, ETSAM-UPM

Irma Arribas Pérez

Dra. Arquitecta, Diseño, Instituto Europeo de Diseño, IED Barcelona

Raimundo Bambó

Dr. Arquitecto, Urbanismo y ordenación del territorio, EINA-UNIZAR

Iñaki Bergera

Dr. Arquitecto, Proyectos Arquitectónicos, EINA-UNIZAR

Jaume Blancafort

Dr. Arquitecto, Arquitectura y Tecnología de la Edificación, ETSAE-UPCT

Enrique Manuel Blanco Lorenzo

Dr. Arquitecto, Proyectos Arquitectónicos, Urbanismo y Composición, ETSAC-UdC

Francisco Javier Boned Purkiss

Dr. Arquitecto, Composición arquitectónica, eAM'-UMA

Ivan Cabrera i Fausto

Dr. Arquitecto, Mecánica de los Medios Continuos y Teoría de Estructuras, ETSA-UPV

Raúl Castellanos Gómez

Dr. Arquitecto, Proyectos Arquitectónicos, ETSA-UPV

Nuria Castilla Cabanes

Dra. Arquitecta, Construcciones arquitectónicas, ETSA-UPV

David Caralt

Arquitecto, Universidad San Sebastián, Sede Concepción, Chile

Rodrigo Carbajal Ballell

Dr. Arquitecto, Proyectos Arquitectónicos, ETSA-US

Eva Crespo

Dra. Arquitecta, Tecnología de la Arquitectura, ETSAB-UPC

Valentina Cristini

Dra. Arquitecta, Composición Arquitectónica, Instituto de Restauración del Patrimonio, ETSA-UPV

Silvia Colmenares

Dra. Arquitecta, Proyectos Arquitectónicos, ETSAM-UPM

Còssima Cornadó Bardón

Dra. Arquitecta, Tecnología de la Arquitectura, ETSAB-UPC

Eduardo Delgado Orusco

Dr. Arquitecto, Proyectos Arquitectónicos, EINA-UNIZAR

Carmen Díez Medina

Dra. Arquitecta, Composición, EINA-UNIZAR

Débora Domingo Calabuig

Dra. Arquitecta, Proyectos Arquitectónicos, ETSA-UPV 


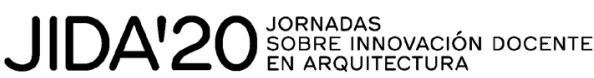

Maria Pia Fontana

Dra. Arquitecta, Arquitectura e Ingeniería de la Construcción, EPS-UdG

Arturo Frediani Sarfati

Dr. Arquitecto, Proyectos, Urbanismo y Dibujo, EAR-URV

Jessica Fuentealba Quilodrán

Arquitecta, Departamento Diseño y Teoría de la Arquitectura, Universidad del Bio-Bío, Concepción, Chile

Pedro García Martínez

Dr. Arquitecto, Arquitectura y Tecnología de la Edificación, ETSAE-UPCT

Mariona Genís Vinyals

Dra. Arquitecta, BAU Centre Universitari de Disseny, UVic-UCC

Eva Gil Lopesino

Arquitecta, Proyectos Arquitectónicos, ETSAM-UPM

María González

Arquitecta, Proyectos Arquitectónicos, ETSA-US

Arianna Guardiola Villora

Dra. Arquitecta, Mecánica de los Medios Continuos y Teoría de Estructuras, ETSA-UPV

Íñigo Lizundia Uranga

Dr. Arquitecto, Construcciones Arquitectónicas, ETSA EHU-UPV

Emma López Bahut

Dra. Arquitecta, Proyectos, Urbanismo y Composición, ETSAC-UdC

Juanjo López de la Cruz

Arquitecto, Proyectos Arquitectónicos, ETSA-US

Luis Machuca Casares

Dr. Arquitecto, Expresión Gráfica Arquitectónica, eAM'-UMA

Magda Mària Serrano

Dra. Arquitecta, Proyectos Arquitectónicos, ETSAV-UPC

Cristina Marieta Gorriti

Dra. Arquitecta, Ingeniería Química y del Medio Ambiente, EIG UPV-EHU

Marta Masdéu Bernat

Dra. Arquitecta, Arquitectura e Ingeniería de la Construcción, EPS-UdG

Camilla Mileto

Dra. Arquitecta, Composición arquitectónica, ETSA-UPV

Zaida Muxí Martínez

Dra. Arquitecta, Urbanismo y ordenación del territorio, ETSAB-UPC

David Navarro Moreno

Dr. Arquitecto, Arquitectura y Tecnología de la Edificación, ETSAE-UPCT

Luz Paz Agras

Dra. Arquitecta, Proyectos, Urbanismo y Composición, ETSAC-UdC

Oriol Pons Valladares

Dr. Arquitecto, Tecnología a la Arquitectura, ETSAB-UPC

Jorge Ramos Jular

Dr. Arquitecto, Teoría de la Arquitectura y Proyectos Arquitectónicos, ETSAVA-UVA

Amadeo Ramos Carranza

Dr. Arquitecto, Proyectos Arquitectónicos, ETSA-US 


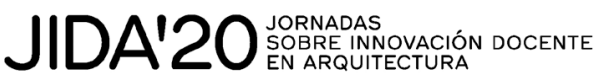

\section{Patricia Reus}

Dra. Arquitecta, Arquitectura y Tecnología de la Edificación, ETSAE-UPCT

\section{Antonio S. Río Vázquez}

Dr. Arquitecto, Composición arquitectónica, ETSAC-UdC

\section{Silvana Rodrigues de Oliveira}

Arquitecta, Proyectos Arquitectónicos, ETSA-US

Carlos Jesús Rosa Jiménez

Dr. Arquitecto, Urbanismo y ordenación del territorio, eAM'-UMA

\section{Jaume Roset Calzada}

Dr. Físico, Física Aplicada, ETSAB-UPC

\section{Patricia Sabín Díaz}

Dra. Arquitecta, Proyectos Arquitectónicos, Urbanismo y Composición, ETSAC-UdC

\section{Mara Sánchez Llorens}

Dra. Arquitecta, Ideación Gráfica Arquitectónica, ETSAM-UPM

\section{Carla Sentieri Omarrementeria}

Dra. Arquitecta, Proyectos Arquitectónicos, ETSA-UPV

\section{Marta Serra Permanyer}

Dra. Arquitecta, Teoría e Historia de la Arquitectura y Técnicas de la Comunicación, ETSAV-UPC

Sergio Vega Sánchez

Dr. Arquitecto, Construcción y Tecnologías Arquitectónicas, ETSAM-UPM

José Vela Castillo

Dr. Arquitecto, Culture and Theory in Architecture and Idea and Form, IE School of Architecture and Design, IE University, Segovia

\section{Isabel Zaragoza de Pedro}

Dra. Arquitecta, Representación Arquitectónica, ETSAB-UPC 


\section{ÍNDICE}

1. Coronawar. La docencia como espacio de resistencia. Coronawar. Teaching as a space of resistance. Ruiz-Plaza, Angela; De Coca-Leicher, José; Torrego-Gómez, Daniel.

2. Narrativa gráfica: el aprendizaje comunicativo del dibujar. Graphic narrative: the communicative learning of drawing. Salgado de la Rosa, María Asunción; Raposo Grau, Javier Fcob; Butragueño Díaz-Guerra, Belén.

3. Sobre la casa desde casa: nueva experiencia docente en la asignatura Taller de Arquitectura. About the house from home: new teaching experience in the subject Architecture Workshop. Millán-Millán, Pablo Manuel.

4. Muéstrame Málaga: Un recorrido por la historia de la arquitectura guiado por el alumnado. Show me Malaga: A tour through the history of architecture guided by students. González-Vera, Víctor Miguel.

5. Formaciones Feedback. Tres proyectos con materiales granulares manipulados robóticamente. Feedback Formation. Three teaching projects on robotically manipulated granular materials. Medina-lbáñez, Jesús; Jenny, David; Gramazio, Fabio; Kohler, Matthias.

6. La novia del Maule, recreación a escala 1:1. The Maule's Bride, recreation 1:1 scale. Zúñiga-Alegría, Blanca.

7. Docencia presencial con evaluación virtual. La adaptación del sistema de evaluación. On-site teaching with on-line testing. The adaptation of the evaluation system. Navarro-Moreno, David; La Spina, Vincenzina; Garcia-Martínez, Pedro; Jiménez-Vicario, Pedro.

8. El uso de rompecabezas en la enseñanza de la historia urbana. The use of puzzles in the teaching of urban history. Esteller-Agustí, Alfred; Vigil-de Insausti, Adolfo; Herrera-Piñuelas, Isamar Anicia.

9. Estrategias educativas innovadoras para la docencia teórica en Arquitectura. Innovative Educational Strategies for Theoretical Teaching in Architecture. LopezDe Asiain, María; Díaz-García, Vicente.

10. Los retos de la evaluación online en el aprendizaje universitario de la arquitectura. Challenges of online evaluation in the Architecture University learning. Onecha-Pérez, Belén; López-Valdés, Daniel; Sanz-Prat, Javier. 
11. Zoé entra en casa. La biología en la formación en arquitectura. Zoé enters the house. Biology in architectural training. Tapia Martín, Carlos; Medina Morillas, Carlos.

12. Elementos clave de una base sólida que estructure la docencia de arquitectura. Key elements of a solid foundation that structures architectural teaching. Santalla-Blanco, Luis Manuel.

13. Buildings $360^{\circ}$ : un nuevo enfoque para la enseñanza en construcción. Buildings 360 : a new approach to teaching construction. Sánchez-Aparicio, Luis Javier; Sánchez-Guevara Sánchez, María del Carmen; Gallego Sánchez-Torija, Jorge; Olivieri, Francesca.

14. Asignaturas tecnológicas en Arquitectura en el confinamiento: hacia una enseñanza aplicada. Technological courses in Architecture during lock down: towards an applied teaching. Cornadó, Còssima; Crespo, Eva; Martín, Estefanía.

15. Pedagogía colaborativa y redes sociales. Diseñar en cuarentena. Collaborative Pedagogy and Social Networks. Design in Quarantine. Hernández-Falagán, David.

16. De Vitruvio a Instagram: Nuevas metodologías de análisis arquitectónico. From Vitruvius to Instagram: New methodologies for architectural análisis. Coeffé Boitano, Beatriz.

17. Estrategias transversales. El grano y la paja. Transversal strategies. Wheat and chaff. Alfaya, Luciano; Armada, Carmen.

18. Lo fortuito como catalizador para el desarrollo de una mentalidad de crecimiento. Chance as a catalyst for the development of a growth mindset. Amtmann-Barbará, Sebastián; Mosquera-González, Javier.

19. Sevilla: Ciudad Doméstica. Experimentación y Crítica Urbana desde el Confinamiento. Sevilla: Domestic City. Experimentation and Urban Critic from Confinement. Carrascal-Pérez, María F.; Aguilar-Alejandre, María.

20. Proyectos con Hormigón Visto. Repensar la materialidad en tiempos de COVID19. Architectural Design with Exposed Concrete. Rethinking materiality in times of COVID-19. Lizondo-Sevilla, Laura; Bosch-Roig, Luis.

21. El Database Driven Lab como modelo pedagógico. Database Driven Lab as a pedagogical model. Juan-Liñán, Lluís; Rojo-de-Castro, Luis.

22. Taller de visitas de obra, modo virtual por suspensión de docencia presencial. Building site visits workshop, virtual mode for suspension of in-class teaching. Pinilla-Melo, Javier; Aira, José-Ramón; Olivieri, Lorenzo; Barbero-Barrera, María del Mar. 
23. La precisión en la elección y desarrollo de los trabajos fin de máster para una inserción laboral efectiva. Precision in the choice and development of the final master's thesis for effective job placement. Tapia-Martín, Carlos; Minguet-Medina, Jorge.

24. Historia de las mujeres en la arquitectura. 50 años de investigación para un nuevo espacio docente. Women's History in Architecture. 50 years of reseach for a new teaching area. Pérez-Moreno, Lucía C.

25. Sobre filtros aumentados transhumanos. HYPERFILTER, una pedagogía para la acción FOMO. On transhuman augmented filters. HYPERFILTER, a pedagogy for FOMO Action. Roig, Eduardo.

26. El arquitecto ante el nuevo paradigma del paisaje: implicaciones docentes. The architect addressing the new landscape paradigm: teaching implications. LópezSanchez, Marina; Linares-Gómez, Mercedes; Tejedor-Cabrera, Antonio.

27. 'Arquigramers'. 'Archigramers'. Flores-Soto, José Antonio.

28. Poliesferas Pedagógicas. Estudio analítico de las cosmologías locales del Covid19. Pedagogical Polysoheres. Analytical study of the local cosmologies of the Covid-19. Espegel-Alonso, Carmen; Feliz-Ricoy, Sálvora; Buedo-García, Juan Andrés.

29. Académicas enREDadas en cuarentena. Academic mamas NETworking in quarantine. Navarro-Astor, Elena; Guardiola-Víllora, Arianna.

30. Aptitudes de juicio estético y visión espacial en alumnos de arquitectura. Aesthetic judgment skills and spatial vision in architecture students. Iñarra-Abad, Susana; Sender-Contell, Marina; Pérez de los Cobos-Casinello, Marta.

31. La docencia en Arquitectura desde la comprensión tipológica compositiva. Teaching Architecture from a compositive and typological understanding. Cimadomo, Guido.

32. Habitar el confinamiento: una lectura a través de la fotografía y la danza contemporánea. Inhabiting confinement: an interpretation through photography and contemporary dance. Cimadomo, Guido.

33. Docencia Conversacional. Conversational learning. Barrientos-Turrión, Laura.

34. ¿Arquitectura a distancia? Comparando las docencias remota y presencial en Urbanismo. Distance Learning in Architecture? Online vs. On-Campus Teaching in Urbanism Courses. Ruiz-Apilánez, Borja; García-Camacha, Irene; Solís, Eloy; Ureña, José María de. 
35. El taller de paisaje, estrategias y objetivos, empatia, la arquitectura como respuesta. The landscape workshop, strategies and objectives, empathy, architecture as the answer. Jiliberto-Herrera, José Luís.

36. Yo, tú, nosotras y el tiempo en el espacio habitado. Me, you, us and time in the inhabited space. Morales-Soler, Eva; Minguet-Medina, Jorge.

37. Mis climas cotidianos. Didácticas para una arquitectura que cuida el clima y a las personas. Climates of everyday life. Didactics for an Architecture that cares for the climate and people. Alba-Pérez-Rendón, Cristina; Morales-Soler, Eva; MartínRuíz, Isabel.

38. Aprendizaje confinado: Oportunidades y percepción de los estudiantes. Confined learning: Opportunities and perception of college students. Redondo-Pérez, María; Muñoz-Cosme, Alfonso.

39. Arqui-enología online. La arquitectura de la percepción, los sentidos y la energía. Archi-Oenology online. The architecture of senses, sensibilities and energies. Ruiz-Plaza, Angela.

40. La piel de Samantha: presencia y espacio. Propuesta de innovación docente en Diseño. The skin of Samantha: presence and space. Teaching innovation proposal in Design. Fernández-Barranco, Alicia.

41. El análisis de proyectos como aprendizaje transversal en Diseño de Interiores. Analysis of projects as a transversal learning in Interior Design. González-Vera, Víctor Miguel; Fernández-Contreras, Raúl; Chamizo-Nieto, Francisco José.

42. El dibujo como herramienta operativa. Drawing as an operational tool. Bacchiarello, María Fiorella.

43. Experimentación con capas tangibles e intan-gibles: COVID-19 como una capa intangible más. Experimenting with tangible and intangible layers: COVID-19 as another intangible layer. Sádaba, Juan; Lenzi, Sara; Latasa, Itxaro.

44. Logros y Límites para una enseñanza basada en el Aprendizaje en Servicio y la Responsabilidad Social Universitaria. Achievements and Limits for teaching based on Service Learning and University Social Responsibility. Ríos-Mantilla, Renato; Trovato, Graziella.

45. Generación screen: habitar en tiempos de confinamiento. Screen Generation: Living in the Time of Confinement. De-Gispert-Hernández, Jordi; García-Ortega, Ramón.

46. Sobre el QUIÉN en la enseñanza arquitectónica. About WHO in architectural education. González-Bandera, María Isabel; Alba-Dorado, María Isabel. 
47. La docencia del dibujo arquitectónico en época de pandemia. Teaching architectural drawing in times of pandemic. Escoda-Pastor, Carmen; Sastre-Sastre, Ramon; Bruscato-Miotto Underlea.

48. Aprendizaje colaborativo en contextos postindustriales: catálogos, series y ensamblajes. Collaborative learning in the post-industrial context: catalogues, series and assemblies. de Abajo Castrillo, Begoña; Espinosa Pérez, Enrique; GarcíaSetién Terol, Diego; Ribot Manzano, Almudena.

49. El Taller de materia. Creatividad en torno al comportamiento estructural. Matter workshop. Creativity around structural behavior. Arias Madero, Javier; Llorente Álvarez, Alfredo.

50. Human 3.0: una reinterpretación contemporánea del Ballet Triádico de Oskar Schlemmer. Human 3.0: a contemporary reinterpretation of Oskar Schlemmer's Triadic Ballet. Tabera Roldán, Andrés; Vidaurre-Arbizu, Marina; Zuazua-Ros, Amaia; González-Gracia, Daniel.

51. ¿Materia o bit? Maqueta real o virtual como herramienta del Taller Integrado de Proyectos. Real or Virtual Model as an Integrative Design Studio Tool. TárragoMingo, Jorge; Martín-Gómez, César; Santas-Torres, Asier; Azcárate-Gómez, César.

52. Un estudio comparado. Hacía la implantación de un modelo docente mixto. $A$ comparative study. Towards the implementation of a mixed teaching model. Pizarro Juanas, María José; Ruiz-Pardo, Marcelo; Ramírez Sanjuán, Paloma.

53. De la clase-basílica al mapa generativo: Las redes colaborativas del nativo digital. From the traditional classroom to the generative map: The collaborative networks of the digital native. Martínez-Alonso, Javier; Montoya-Saiz, Paula.

54. Confinamiento liberador: experimentar con materiales y texturas. Liberating confinement: experimenting with materials and textures. De-Gispert-Hernández, Jordi.

55. Exposiciones docentes. Didáctica, transferencia e innovación en el ámbito académico. Educational exhibitions. Didacticism, transfer and innovation into the academic field. Domigo Santos, Juana; Moreno Álvarez, Carmen; García Píriz, Tomás.

56. Comunicacción. Acción formativa sobre la comunicación efectiva. Communicaction. Training action about the effective communication. Rivera, Rafael; Trujillo, Macarena.

57. Oscilación entre teoría y práctica: la representación como punto de equilibrio. Oscillation between theory and practice: representation as a point of balance. Andrade-Harrison, Pablo. 


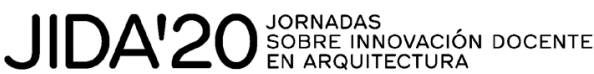

58. Construcción de Sentido: Rima de Teoría y Práctica en el Primer Año de Arquitectura. Construction of Meaning: Rhyme of Theory and Practice in the First Year of Architecture. Quintanilla-Chala, José; Razeto-Cáceres, Valeria.

59. Propuesta innovadora en el Máster Oficial en Peritación y Reparación de Edificios. Innovative proposal in the Official Master in Diagnosis and Repair of Buildings. Pedreño-Rojas, Manuel Alejandro; Pérez-Gálvez, Filomena; MoralesConde, María Jesús; Rubio-de-Hita, Paloma.

60. La inexistencia de enunciado como enunciado. The nonexistence of statement as statement. García-Bujalance, Susana.

61. Blended Learning en la Enseñanza de Proyectos Arquitectónicos a través de Miro. Blended Learning in Architectural Design Education through Miro. CoelloTorres, Claudia.

62. Multi-Player City. La producción de la ciudad negociada: Simulaciones Docentes. Multi-Player City. The production of the negociated city: Educational Simulations. Arenas Laorga, Enrique; Basabe Montalvo, Luis; Muñoz Torija, Silvia; Palacios Labrador, Luis.

63. Proyectando un territorio Expo: grupos mixtos engarzando el evento con la ciudad existente. Designing an Expo space: mixed level groups linking the event with the existing city. Gavilanes-Vélaz-de-Medrano, Juan; Castellano-Pulido, Javier; Fuente-Moreno, Jesús; Torre-Fragoso, Ciro.

64. Un pueblo imaginado. An imagined village. Toldrà-Domingo, Josep Maria; FarrenyMorancho, Jaume; Casals-Roca, Raquel; Ferré-Pueyo, Gemma.

65. El concurso como estrategia de aprendizaje: coordinación, colaboración y difusión. The contest as a learning strategy: coordination, collaboration and dissemination. Fernández Villalobos, Nieves; Rodríguez Fernández, Carlos; Geijo Barrientos, José Manuel.

66. Aprendizaje-Servicio para la diagnosis socio-espacial de la edificación residencial. Service-Learning experience for the socio-spatial diagnosis of residential buildings. Vima-Grau, Sara; Tous-Monedero, Victoria; Garcia-Almirall, Pilar.

67. Creatividad con método. Evolución de los talle-res de Urbanismo+Proyectos de segundo curso. Creativity within method. Evolution of the se-cond year Architecture+Urban design Studios. Frediani Sarfati, Arturo; Alcaina Pozo, Lara; Rius Ruiz, Maria; Rosell Gratacòs, Quim.

68. Estrategias de integración de la metodología BIM en el sector AEC desde la Universidad. Integration strategies of the BIM methodology in the AEC sector from the University. García-Granja, María Jesús; de la Torre-Fragoso, Ciro; BlázquezParra, Elidia B.; Martin-Dorta, Norena. 
69. Taller experimental de arquitectura y paisaje. Primer ensayo "on line". Architecture and landscape experimental atelier. First online trial. Coca-Leicher, José de; Fontcuberta-Rueda, Luis de.

70. camp_us: co-diseñando universidad y ciudad. Pamplona, 2020. camp_us: codesigning university and city. Pamplona 2020. Acilu, Aitor; Larripa, Adrián.

71. Convertir la experiencia en experimento: La vida confinada como escuela de futuro. Making the experience into experiment: daily lockdown life as a school for the future. Nanclares-daVeiga, Alberto.

72. Urbanismo Acción: Enfoque Sostenible aplicado a la movilidad urbana en centros históricos. Urbanism Action: Sustainable Approach applied to urban mobility in historic centers. Manchego-Huaquipaco, Edith Gabriela; Butrón-Revilla, Cinthya Lady.

73. Arquitectura Descalza: proyectar y construir en contextos frágiles y complejos. Barefoot Architecture designing and building in fragile and complex contexts. López-Osorio, José Manuel; Muñoz-González, Carmen M.; Ruiz-Jaramillo, Jonathan; Gutiérrez-Martín, Alfonso.

74. I Concurso de fotografía de ventilación y climatización: Una experiencia en Instagram. I photography contest of ventilation and climatization: An experience on Instagram. Assiego-de-Larriva, Rafael; Rodríguez-Ruiz, Nazaret.

75. Urbanismo participativo para la docencia sobre espacio público, llegó el confinamiento. Participatory urbanism for teaching on public space, the confinement arrived. Telleria-Andueza, Koldo; Otamendi-Irizar, Irati.

76. WhatsApp: Situaciones y Programa. WhatsApp: Situations and Program. Silva, Ernesto; Braghini, Anna; Montero Paulina.

77. Los talleres de experimentación en la formación del arquitecto humanista. The experimental workshops in the training of the humanist architect. DomènechRodríguez, Marta; López López, David.

78. Role-Play como Estrategia Docente en el Aprendizaje de la Construcción. RolePlay as a Teaching Strategy in Construction Learning. Pérez-Gálvez, Filomena; Pedreño-Rojas, Manuel Alejandro; Morales-Conde, María Jesús; Rubio-de-Hita, Paloma.

79. Enseñanza de la arquitectura en Chile. Acciones pedagógicas con potencial innovador. Architectural teaching in Chile. Pedagogical actions with innovative potential. Lagos-Vergara, Rodrigo; Barrientos-Díaz, Macarena. 
80. Taller vertical y juego de roles en el aprendizaje de programas arquitectónicos emergentes. Vertical workshop and role-playing in the learning of emerging architectural programs. Castellano-Pulido, F. Javier; Gavilanes-Vélaz de Medrano, Juan; Minguet-Medina, Jorge; Carrasco-Rodríguez, Francisco.

81. Un extraño caso de árbol tenedor. Madrid y Ahmedabad. Aula coopera [Spain/in/India]. A curious case of tree fork. Madrid and Ahmedabad. Aula coopera [Spain/in/India]. Montoro-Coso, Ricardo; Sonntag, Franca Alexandra.

82. La escala líquida. Del detalle al territorio como herramienta de aprendizaje. Liquid scale. From detail to territory as a learning tool. Solé-Gras, Josep Maria; TiñenaRamos, Arnau; Sardà-Ferran, Jordi.

83. Empatía a través del juego. La teoría de piezas sueltas en el proceso de diseño. Empathy through playing. The theory of loose parts in Design Thinking. CabreroOlmos, Raquel.

84. La docencia de la arquitectura durante el confinamiento. El caso de la Escuela de Valencia. Teaching architecture in the time of stay-at-home order. The case of the Valencia School. Cabrera i Fausto, Ivan; Fenollosa Forner, Ernesto.

85. Proyectos Arquitectónicos de programa abierto en lugares invisibles. Architectural Projects of open program in invisible places. Alonso-García, Eusebio; Blanco-Martín, Javier. 


\title{
Logros y Límites para una enseñanza basada en el Aprendizaje en Servicio y la Responsabilidad Social Universitaria
}

\section{Achievements and Limits for teaching based on Service Learning and University Social Responsibility}

Ríos-Mantilla, Renato; Trovato, Graziellab

a Doctorando por la UPM (DOCA) y profesor por la PUCE (FADA, Quito, Ecuador) r.riosm@alumnos.upm.es; rsrios@puce.edu.ec; ${ }^{b}$ Secretaria académica en el DOCA (UPM), Doctora por la UPM graziella.trovato@upm.es

\begin{abstract}
The following article shows the importance of University Social Responsibility (USR) in Latin America, especially in the city of Quito-Ecuador. The research aims to show a general overview of USR and its implementation in the context, analyze its strengths and weaknesses to propose long-term synergies that allow strengthening the system, generating debates and achieving greater impacts that are in accordance with the development plans of each country. For a critical analysis, a look from the outside is necessary to see the coherence of the activities carried out. Although it is true that USR seeks to highlight the social aspect, it cannot be separated from human rights and environmental justice approaches. The study cases demonstrates different approaches to service learning (SL) in the architecture career and its articulation with each university position of the USR.
\end{abstract}

Keywords: university-social-responsibility, training, public-space, participatory-architecture.

Thematic areas: architectural projects, urban planning and spatial planning, servicelearning, humanitarian architecture.

\begin{abstract}
Resumen
El siguiente artículo muestra la importancia de la Responsabilidad Social Universitaria (RSU) en el ámbito Latinoamericano, en especial, en la ciudad de Quito-Ecuador. La investigación pretende mostrar un panorama general de la RSU y su implementación en el contexto, analizar sus fortalezas y sus fragilidades para proponer sinergias a largo plazo que permitan robustecer el sistema, generar debates y alcanzar mayores impactos que estén acorde a los planes de desarrollo de cada país. Para un análisis crítico es necesaria una mirada desde fuera para ver la coherencia de las actividades realizadas. Si bien es cierto que la RSU busca destacar el aspecto social, éste no puede ser desligado de enfoques de derechos humanos y justicia medio ambiental. Los cuatro casos de estudio demuestran diversos enfoques de aprendizaje en servicio (ApS) en la carrera de arquitectura y su articulación con cada postura universitaria de la RSU.
\end{abstract}

Palabras clave: responsabilidad-social-universitaria, formación, espacio-público, arquitectura-participativa.

Bloque temático: proyectos arquitectónicos y urbanos, aprendizaje-servicio, arquitectura humanitaria. 


\section{La RSU en América Latina: hacia el carácter público de la educación}

Desde la declaración mundial de la UNESCO de 1998 hacia la educación superior, la responsabilidad universitaria se ha ido perfilando como un ámbito necesario de compromiso de las instituciones. En el caso de las Universidades se define claramente su papel fundamental en el desarrollo de las comunidades, y, se ponen en debate las consecuencias éticas y las responsabilidades que deben cumplir con el entorno para alcanzar un futuro sostenible; dando pauta para la creación de los Objetivos de Desarrollo Sostenible del milenio (ODS) y para los lineamientos de Responsabilidad Social (RS) en sus diferentes ámbitos: empresariales, universitarios, corporativos, etc.

A lo largo de estos años, como es conocido, se han creado redes específicas para su implementación y si bien existe una divulgación de resultados, suele proceder de los profesionales implicados en primera persona, desde su experiencia, echándose en falta una valoración crítica externa que compare metodologías y resultados. En Latinoamérica las tres redes principales que tratan los temas de Responsabilidad Social Universitaria (RSU) son: ORSU (Observatorio de Responsabilidad Social Universitaria de Colombia), AUSJAL (Asociación de Universidades Confiadas a la Compañía de Jesús en América Latina), y URSULA (Unión de Responsabilidad Social Universitaria Latinoamericana). Además, el Banco Interamericano de Desarrollo (BID) (2009) ha creado un manual que guía a las universidades para la aplicación de la RS. Balance entre aportación pública, privada y privada - jesuita Por otro lado, la editorial Netbiblo ha publicado el libro: "Responsabilidad Social Universitaria" en el cual a través de varios autores de diversa proveniencia en el ámbito iberoamericano, demuestra la importancia de la academia en la formación de profesionales con valores ciudadanos (2010). Desde la práctica, se ha observado que el Aprendizaje en Servicio (ApS) es la metodología educativa que ha acercado los lineamientos de RSU al campo, siendo el Centro Latinoamericano de Aprendizaje en Servicio Solidario (CLAYSS) el más fuerte en la región.

De los ejemplos citados es posible delinear los objetivos compartidos así como ciertas cuestiones metodológicas comunes. Destacamos:

Cooperar en lugar de competir.

La Responsabilidad Social es repensar el carácter público de la Educación, es decir, cómo la Universidad puede solventar las expectativas que genera en su contexto, incluso siendo a veces una institución privada, aportando a que la mayoría de los habitantes tengan las mismas oportunidades para desarrollarse, eliminando obstáculos sociales, culturales, económicos y políticos (Gasca - Pliego \& Olvera - García, 2011). Por medio de este pensamiento se fortalecen las decisiones colectivas, fomentando a los ciudadanos críticos y partícipes de su propia transformación, siendo parte de un proyecto en común. Para José Beltrán y otros autores (2014) es fundamental repensar y diagnosticar la docencia actualmente, suprimiendo ámbitos de competición e incentivando a la cooperación, donde el compromiso social debe ser la guía para los planes de mejoramiento.

La importancia del proceso y la transparencia de los resultados.

Este tipo de enfoques obliga a las Universidades a una mejora continua, donde la rendición de cuentas y la transparencia permite evidenciar los avances realizados en cada paso propuesto; para Demin (1989) se debe realizar un ciclo de: Actuar, Planear, Hacer y Verificar, para asegurar la efectividad los procesos. 


\subsection{Necesidad de una mirada crítica comparativa: el caso de Ecuador}

En Latinoamérica estos conceptos han llegado a las Universidades para cambiar la forma de educar y acercarla a la realidad, articulando Investigación, Vinculación con la Colectividad, y Docencia, respondiendo con cada una a las necesidades de la población, además, de repensar las acciones de Gestión. En Ecuador el Consejo de Educación Superior (CES) desde el 2013, en su Reglamento de Régimen Académico (2019), artículo 4, establece que la Vinculación con la Colectividad debe generar respuestas a las necesidades de su entorno, respondiendo con capacidades "técnicas" al territorio. De esta forma, se impulsó al desarrollo de propuestas y estrategias para acercar a las Universidades al territorio, restaurando tejidos sociales deteriorados y aportando a las necesidades de la población.

Otra particularidad del Ecuador es que entre el 2013 y 2017 el Plan Nacional de Desarrollo (PND) se orientó hacia el concepto andino del "Buen Vivir" por lo que contempló 12 objetivos nacionales que buscaban un Estado incluyente, solidario y democrático, incentivando al trabajo con comunidades vulnerables1. Para el período 2017 - 2021 el PND se reorientó con 9 objetivos vinculados a: Derechos, Economía y Gobernanza, "Toda una Vida". De esta forma el Estado coloca a la Academia en un rol más activo en los procesos de cambio de la población.

Como casos de estudio, la investigación se centró en el trabajo realizado por cuatro Universidades de la ciudad de Quito, realizando entrevistas con los responsables de Vinculación de las carreras de arquitectura para conocer el estado de la RSU en las universidades, comparar métodos y plantear redes que acerquen a la carrera y al ciudadano.

\subsection{Responsabilidad Social y definición del perfil profesional del arquitecto}

Según el diario el Comercio de Quito (2017) el 60\% de las edificaciones son realizadas de manera informal en la ciudad, evidenciando la grave situación de las construcciones en casos de desastres naturales; y por otro lado, también, muestra la poca intervención profesional en el territorio, dejando claro que no existen políticas públicas fuertes que logren solucionar los problemas de precariedad de la ciudad y que la academia aún no posee una estructura fuerte de apoyo a la ciudadanía. En este punto y con las reformas educativas dadas por el CES se pretende fortalecer la relevancia de la Academia con la sociedad. Además, la investigación cuestionará el papel de la educación en la definición del rol profesional en la arquitectura, ya que en países de Latinoamérica los arquitectos tienen el estigma de ser elitistas sin una postura social. Se desconocen los procesos y su relevancia en el territorio, a veces las Universidades se preocupan más por ganar concursos de arquitectura que analizar y cuestionar el impacto que generan los proyectos en la comunidad.

Desde lo operativo, y para desarrollar la propuesta, el CES ha promovido implementarla desde el área de prácticas pre-profesionales (LOES, 2010), el Reglamento de Régimen Académico determina un número específico de horas como requisito para la titulación, del 2013 al 2019 con 160 horas, y a partir del 2019 con un rango entre 96 a 144 horas, evidenciando la inestabilidad normativa del país. La importancia del estudio está en su mirada crítica, se analizarán los procesos y la coherencia universitaria, además, de analizar fragilidades del sistema que permiten proponer mejoras a largo plazo y fortalecer impactos.

\footnotetext{
${ }^{1}$ Artículo 35 de la Constitución de la República del Ecuador (2008). Art. 35.- Las personas adultas mayores, niñas, niños y adolescentes, mujeres embarazadas, personas con discapacidad, personas privadas de libertad y quienes adolezcan de enfermedades catastróficas o de alta complejidad, recibirán atención prioritaria y especializada en los ámbitos público y privado. La misma recibirán las personas en situación de riesgo, las víctimas de violencia doméstica y sexual, maltrato infantil, desastres naturales o antropogénicos.
} 


\section{Elección de los casos de Estudio: 4 Escuelas de Arquitecturas, 4 modelos de RSU}

En Quito existen ocho2 Universidades que ofertan la carrera de arquitectura: Universidad Central del Ecuador (UCE), Universidad San Francisco de Quito (USFQ), Universidad de las Américas (UDLA), Pontificia Universidad Católica del Ecuador sede Quito (PUCE), Universidad Tecnológica Equinoccial (UTE), Universidad Internacional SEK (SEK), Universidad Internacional del Ecuador (UIDE), y Universidad Amawtay Wasi (Intercultural). Las cuatro primeras abarcan el mayor número de estudiantes y serán las analizadas por sus buenas prácticas de vinculación y por su aportación positiva a las necesidades de la población. La UCE es de carácter público, las otras tres son privadas con diversos enfoques como se explicará más adelante.

Para entender las buenas prácticas se deben analizar procesos que acojan términos de: intergeneracional, intercultural, movilidad, discapacidad, género y derechos de la naturaleza, además, de responder a las necesidades de la comunidad, de aportar en el aspecto formativo, e incidir en una conciencia social (experiencia significativa) (RSU AUSJAL , 2019). En los casos de estudio se realizaron cinco entrevistas entre diciembre de 2019 y enero de 2020, se recopilaron datos de misión y visión de siete de las ocho carreras de arquitectura de Quito y se elaboró una guía para enfatizar los aspectos de RSU.

\subsection{La UCE: reciprocidad entre formación y necesidades sociales del entorno}

La UCE se fundó en 1620 y posee aproximadamente 50.000 estudiantes (UCE, 2020). La entrevista se la realizó el 4 de diciembre de 2019 al arquitecto Carlos Hidalgo, responsable de Vinculación de la carrera de arquitectura. La Universidad posee un departamento de Vinculación general que promueve el trabajo transdisciplinar. Desde arquitectura se busca un enfoque macro - territorial, que permita aportar a los Planes de Desarrollo de las Comunidades (Hidalgo, 2019). Se trabaja desde la investigación aplicada, además, de fomentar cursos y servicios al público exterior.

Como buena práctica UCE se observa el trabajo realizado por el OPTE, Observatorio de la Producción del Territorio Ecuatoriano, en el que se reflexionan sobre las circunstancias nacionales y locales del país, articulando videos y narrativas en sus análisis. Generan diagnósticos que ayudan a comprender la realidad del país.

\subsection{La UDLA. Un modelo privado de Aprendizaje en Servicio: desarrollo local desde sinergias institucionales}

La UDLA es una universidad privada fundada en 1995 como franquicia internacional y posee alrededor de 17.000 estudiantes. Sus proyectos de Vinculación se alinean a pilares internacionales de sostenibilidad (UDLA, 2020). La entrevista se la realizó el 10 de diciembre de 2019 en la secretaría de arquitectura, fueron dos entrevistadas, las arquitectas Daniela Maldonado (responsable) y Ana María Rojas (tutora). La parte social de vinculación la dividen en dos partes: la primera como materias comunes y la segunda como proyectos. En el primer caso no tienen una relación directa con la carrera, en el segundo, se trabaja en conjunto con

\footnotetext{
2 página de Cursos y Carreras de Ecuador (2019).
} 
instituciones y se realizan intervenciones de "acupuntura urbana" (Maldonado \& Rojas , 2019). Actualmente buscan reformar materias de Aprendizaje en Servicio.

En arquitectura se ha trabajado en varios territorios, siendo su proyecto emblemático el de "Intervención Interiorista de Centros para niños en situaciones de riesgo", que ha permitido a la carrera trabajar en articulación con varios museos de la ciudad. Los proyectos se alinean dentro de seis programas establecidos que son: "educación"; "crecimiento económico"; "salud y bienestar"; "cultura"; "ambiente"; "industria, innovación e infraestructura" (pilares de la sostenibilidad). En el caso referido, se ha trabajado desde "infraestructura" por el carácter de la propuesta.

\subsection{La USFQ. Un modelo privado para formar emprendedores. La cuestión de las competencias entre Universidad y órdenes profesionales}

La USFQ es una universidad privada fundada en 1988 y con alrededor de 10.000 estudiantes, trabajan por medio de las artes liberales y sus carreras se manejan con materias en común para toda la universidad (USFQ, 2020). Se realizaron dos entrevistas, la primera con el profesor arquitecto Jaime López encargado de extensión de arquitectura, y la segunda con la analista Alejandra Miño que trabaja en la coordinación general de Vinculación. El primer entrevistado explicó que la carrera busca trabajar con proyectos a largo plazo y sin una relación directa con la misma, ya que, según sus lineamientos, entrarían en una competencia desleal con el gremio.; pueden apoyar tangencialmente, y, luego obligatoriamente las comunidades deben buscar a un profesional para concluir con la propuesta (López , 2019). En la segunda entrevista se explicaron los procesos de forma macro y que la responsabilidad que se tiene es más hacia la formación de los estudiantes (Miño, 2020) y no tanto hacia las necesidades externas.

Desde Arquitectura se han liderado tres proyectos: dos en la provincia de Chimborazo, "Rediseñando textiles" de Guano y "Desarrollo de estudios para la restauración de residencia patrimonial" en Alausí, el tercero en Quito, "Niños seguros en las vías del Ecuador". Se trabaja indirectamente con competencias técnicas, es decir, con mapas, análisis, con elementos previos a la definición de un diseño que son entregados a la comunidad; si la misma requiere continuar entrará a la forma de servicios. Su enfoque está en potenciar las habilidades blandas de los estudiantes como: colaboración, adaptabilidad, manejo del tiempo, etc.

\subsection{La PUCE. EI diseño participativo: formando "arquitectos intérpretes de sus realidades"}

La PUCE tiene una formación ignaciana y fue fundada en 1946. La sede Quito tiene un promedio de 14.500 estudiantes; su misión y visión se basan en principios humanos donde el rol social es importante en la enseñanza y aprendizaje (PUCE, 2020). En este caso no se realizó entrevista, sino que se recopiló información sobre los elementos conceptuales que permiten entender el trabajo de vinculación, siendo lo social trabajado desde servicio comunitario (PUCE, 2020). Los conceptos de responsabilidad son basados en el modelo AUSJAL. Dentro de arquitectura se maneja la idea de trabajar "con la comunidad" para llegar a diálogos y consensos (Ríos Mantilla , González, Armijos , \& Otros, 2016). Además, se trabaja desde talleres y materias que complementan la reflexión sobre el trabajo realizado socialmente. Lo que se busca es mejorar la calidad de vida de los pueblos (González, Yépez , \& García , 2019), reforzando temas de interculturalidad e identidad. 
El programa de arquitectura, "Arquitectura con la Comunidad", trabaja desde tres aristas: anteproyectos, talleres, e intervenciones espaciales que buscan fortalecer a las comunidades, convirtiendo a las iniciativas en herramientas para transformar la realidad. También existe el "Laboratorio de los Paisajes Vivos" donde se analiza al paisaje desde diferentes ámbitos y aproximaciones. Y desde la docencia existe la clase de Gestión Comunitaria que busca reflexionar sobre las metodologías de gestión; entre otros ejemplos.

\subsection{Análisis comparativo}

Desde la enseñanza pública se observa una visión más urbano - territorial, mientras que en la privada se ve más una postura interior de "acupuntura urbana", donde por medio de intervenciones a menor escala se generan cambios en las comunidades. Las cuatro universidades poseen direcciones generales de vinculación que centralizan y dan los lineamientos de procedimientos. Por otro lado, se puede observar que desde lo privado cada universidad le da su enfoque propio ya sea: comercial, empresarial o humano.

A manera de comparación y análisis se puede determinar que en la UCE reviste una fuerte importancia la relación de los proyectos con la realidad social del país, especialmente vista desde lo técnico - constructivo, con apoyo a los Planes de Desarrollo del país (Hidalgo, 2019). En la UDLA y USFQ se puede observar una mirada más interior, centrada en el estudiante, ya que se busca fortalecer las habilidades blandas3; se enfocan desde la prestación de servicios con un apoyo tangencial a las comunidades, y priorizando la experiencia de los alumnos. Desde la PUCE se tiene una relación interior - exterior que permite entender las problemáticas desde una mirada de ponerse en los zapatos del otro para generar una respuesta; los proyectos se manejan desde las necesidades comunitarias de una forma técnica y desde la sensibilización de la profesión (Ríos Mantilla, González, Armijos , \& Otros, 2016).

Para poder realizar las entrevistas, recopilación de información y posterior análisis comparativo, se establecieron diez parámetros: Datos Generales, Metodología, RSU, Impactos, Buenas Prácticas, Ejemplos, Territorios, Hojas de Ruta, Estrategias y Articulaciones. Sobre esta base se ha elaborado la siguiente tabla comparativa que nos permite entender las diferencias y semejanzas de trabajo de vinculación en las Universidades, establecer puntos de articulación, y la forma de aplicación de la RSU y el ApS en las carreras de arquitectura en Quito.

\footnotetext{
${ }^{3}$ Como el trabajo en equipo, puntualidad, mejorar la comunicación y proactividad.
} 
Tabla 1. Tabla Compartaiva

\begin{tabular}{|c|c|c|c|c|c|}
\hline \multicolumn{2}{|c|}{$\begin{array}{l}\text { UNIVERSIDAD I } \\
\text { PARÁMETRO }\end{array}$} & \multirow{2}{*}{$\begin{array}{l}\text { UCE } \\
\text { Fundada } 1620 . \\
50.000 \text { alumnos. } \\
\text { Misión y Visión } \\
\text { relacionadas con el } \\
\text { entorno. } \\
\text { Pública. }\end{array}$} & \multirow{2}{*}{\begin{tabular}{l}
\multicolumn{1}{c}{ UDLA } \\
Fundada en 1995. \\
17.000 alumnos. \\
Sinergias \\
institucionales \\
(empresa). \\
Privada.
\end{tabular}} & \multirow{2}{*}{\begin{tabular}{l}
\multicolumn{1}{c}{ USFQ } \\
Fundada en 1988. \\
10.000 alumnos. \\
Librepensadores, \\
emprendedores, \\
artes liberales. \\
Privada.
\end{tabular}} & \multirow{2}{*}{$\begin{array}{l}\text { PUCE } \\
\text { Fundada en } 1946 . \\
14.500 \text { alumnos. } \\
\text { Formación Ignaciana, } \\
\text { principios humanos y } \\
\text { rol social. Privada. }\end{array}$} \\
\hline 1. & $\begin{array}{l}\text { Datos } \\
\text { Generales }\end{array}$ & & & & \\
\hline 2. & Metodología & $\begin{array}{l}\text { Investigación - } \\
\text { Acción. Se trabaja } \\
\text { desde necesidades. } \\
\text { Más urbano. }\end{array}$ & $\begin{array}{l}\text { Diseño Participativo } \\
\text { - Diagnóstico, } \\
\text { diseño y resultados. }\end{array}$ & $\begin{array}{l}\text { Variables se buscan } \\
\text { proyectos a largo } \\
\text { plazo. }\end{array}$ & $\begin{array}{l}\text { Diseño Participativo - } \\
\text { Arquitectura CON la } \\
\text { Comunidad. }\end{array}$ \\
\hline 3. & RSU & $\begin{array}{l}\text { Sensibilización } \\
\text { mediante } \\
\text { capacitaciones y } \\
\text { cursos. }\end{array}$ & $\begin{array}{l}\text { Modelo de } \\
\text { colaboración y } \\
\text { apoyo. }\end{array}$ & $\begin{array}{l}\text { Enfocada en } \\
\text { procesos de gestión. } \\
\text { Modelo Empresarial. }\end{array}$ & $\begin{array}{l}\text { Basada en revalorizar } \\
\text { la identidad de los } \\
\text { pueblos. AUSJAL }\end{array}$ \\
\hline 4. & Impactos & $\begin{array}{l}\text { Más perceptivos y se } \\
\text { apoya desde temas } \\
\text { urbanos que tiene } \\
\text { relación directa con } \\
\text { los planes de } \\
\text { desarrollo. }\end{array}$ & $\begin{array}{l}\text { Aun no se puede } \\
\text { medir, sin embargo, } \\
\text { se ha aportado para } \\
\text { el desarrollo de las } \\
\text { comunidades. }\end{array}$ & $\begin{array}{l}\text { Más cuantitativo en } \\
\text { relación acciones y } \\
\text { procesos realizados. }\end{array}$ & $\begin{array}{l}\text { Un alto grado de } \\
\text { satisfacción. Se ha } \\
\text { logrado una } \\
\text { conciencia social y } \\
\text { reconocer las } \\
\text { necesidades de la } \\
\text { población. }\end{array}$ \\
\hline 5. & $\begin{array}{l}\text { Buenas } \\
\text { Prácticas }\end{array}$ & $\begin{array}{l}\text { Cuando se articula } \\
\text { con lo académico y lo } \\
\text { social, aportando } \\
\text { desde lo formativo. }\end{array}$ & $\begin{array}{l}\text { Cuando se trabaja } \\
\text { en conjunto con las } \\
\text { poblaciones. }\end{array}$ & $\begin{array}{l}\text { Cuando se actúa } \\
\text { desde ámbitos } \\
\text { gremiales sin } \\
\text { producir } \\
\text { competencia } \\
\text { desleal. }\end{array}$ & $\begin{array}{l}\text { Cuando articulan } \\
\text { ámbitos: educativos, } \\
\text { cognoscitivos, } \\
\text { sociales, } \\
\text { organizacionales, y } \\
\text { ambientales. }\end{array}$ \\
\hline 6. & Ejemplos & $\begin{array}{l}\text { Patologías en Zuleta } \\
\text { y OPTE } \\
\text { (Observatorio de la } \\
\text { Producción del } \\
\text { Territorio } \\
\text { Ecuatoriano). }\end{array}$ & $\begin{array}{l}\text { Intervenciones } \\
\text { interioristas en } \\
\text { museos. }\end{array}$ & $\begin{array}{l}\text { Niños seguros en } \\
\text { las vías, } \\
\text { rediseñando textiles } \\
\text { en Guano, y } \\
\text { Restauración en } \\
\text { Alausí. }\end{array}$ & $\begin{array}{l}\text { Programa de } \\
\text { Arquitectura con la } \\
\text { Comunidad, } \\
\text { Laboratorio de los } \\
\text { Paisajes Vivos, y } \\
\text { Gestión Comunitaria. }\end{array}$ \\
\hline 7. & Territorios & $\begin{array}{l}\text { Noroccidente de } \\
\text { Quito, Zuleta, Mejía, } \\
\text { Mashpi, Cotopaxi. }\end{array}$ & $\begin{array}{l}\text { Quito (MIC, } \\
\text { Guápulo, La } \\
\text { Mariscal, La Bota, } \\
\text { etc.), El Coca. }\end{array}$ & $\begin{array}{l}\text { Guano, Alausí, } \\
\text { Quito. }\end{array}$ & $\begin{array}{l}\text { Esmeraldas, Ibarra, } \\
\text { Manabí, Quito (La } \\
\text { Mariscal, La Loma, La } \\
\text { Merced, etc.), Santo } \\
\text { Domingo, Mejía. }\end{array}$ \\
\hline 8. & Hoja De Ruta & $\begin{array}{l}\text { Carta de pedido, } \\
\text { análisis, convenio, } \\
\text { ficha de trabajo, } \\
\text { informes. }\end{array}$ & $\begin{array}{l}\text { Municipio, } \\
\text { valoración } \\
\text { institucional, } \\
\text { convenio, } \\
\text { diagnóstico, } \\
\text { objetivos, } \\
\text { cronograma, } \\
\text { aprobación. }\end{array}$ & $\begin{array}{l}\text { Acercamiento de la } \\
\text { comunidad, } \\
\text { convenio, análisis, } \\
\text { objetivos, } \\
\text { cronograma, } \\
\text { evidencias, } \\
\text { informes. }\end{array}$ & $\begin{array}{l}\text { Carta de pedido, } \\
\text { respuesta, } \\
\text { cronograma, } \\
\text { diagnóstico, } \\
\text { evidencias, entrega } \\
\text { recepción, informes, } \\
\text { encuestas de } \\
\text { percepción. }\end{array}$ \\
\hline 9. & $\begin{array}{l}\text { Estrategias } \\
\text { Para Trabajar } \\
\text { En EI Territorio }\end{array}$ & $\begin{array}{l}\text { Árbol de problemas, } \\
\text { de soluciones, } \\
\text { talleres lúdicos, } \\
\text { observación, } \\
\text { investigación - } \\
\text { acción. }\end{array}$ & $\begin{array}{l}\text { Talleres con la } \\
\text { comunidad. }\end{array}$ & $\begin{array}{l}\text { Charlas, encuestas, } \\
\text { capacitaciones. }\end{array}$ & $\begin{array}{l}\text { Primeros contactos, } \\
\text { respuestas colectivas, } \\
\text { acciones integrales, } \\
\text { sistematización. } \\
\text { Además, arquitecto } \\
\text { intérprete, respetar } \\
\text { saber del otro, } \\
\text { retroalimentación, } \\
\text { arquitectura con la } \\
\text { comunidad, etc. }\end{array}$ \\
\hline 10. & $\begin{array}{l}\text { Articulaciones } \\
\text { (Investigación } \\
\text { - Docencia - } \\
\text { Vinculación) }\end{array}$ & $\begin{array}{l}\text { Se integra por los } \\
\text { observatorios y por } \\
\text { materias de } \\
\text { proyectos o titulación. }\end{array}$ & $\begin{array}{l}\text { Articulación con } \\
\text { materias, ApS, } \\
\text { investigación. }\end{array}$ & $\begin{array}{l}\text { Mediante ApS e } \\
\text { investigación } \\
\text { aplicada. }\end{array}$ & $\begin{array}{l}\text { Se dan por prácticas, } \\
\text { cursos, eventos, } \\
\text { asesorias, Aps e } \\
\text { investigación aplicada. }\end{array}$ \\
\hline
\end{tabular}

Fuente: Ríos, R. (2019). Base de Datos Universidades Entrevistadas TFM (Universidad Politécnica de Madrid) 


\section{Conclusiones - Fortalezas y Fragilidades en el Sistema}

El análisis comparativo muestra que las Universidades, de forma general, presentan una preocupación sobre el medio exterior y aplican la propuesta de ApS con algunos lineamientos de RSU, a pesar de que sus políticas no son muy conocidas en el país, es decir, la RSU no está trabajada desde un nivel macro, sino solo como un componente de extensión. Como un logro importante en los proyectos se evita el asistencialismo, para no generar dependencia con la contraparte; se busca formar, fortalecer, y transferir el conocimiento para aportar a la población. Comparten en general protocolos burocráticos que entorpecen algunas acciones, poseen una coordinación general que brinda los lineamientos y establece la hoja de ruta a seguir; se empiezan con una carta de pedido o convenio marco, para, posteriormente, realizar diagnósticos, presupuestos, y medición de la satisfacción de las comunidades atendidas.

\section{Objetivos y metodologías}

En temas de diferenciación, la UCE y la PUCE responden más a las poblaciones desde una mirada técnica social, en donde la comunidad o contexto adquieren gran relevancia y el factor humano es muy importante para el desarrollo de la práctica. Por otro lado, la UDLA y la USFQ se centran más en el estudiante y en el desarrollo de sus habilidades blandas, anteriormente definidas. La educación pública tiende a una política exterior más fuerte y la privada hacia la experiencia de los estudiantes. Las metodologías usadas son variadas, fomentando al diseño participativo, sin embargo, la PUCE ha tratado de generar estrategias para entrar en el territorio y darle fuerza a los procesos de vinculación. La UCE se ha desarrollado más en la investigación aplicada. La UDLA y USFQ se han alineado más a estándares internacionales, mientras que la UCE y la PUCE han generado modelos de identidad propios.

\section{Fragilidades del sistema}

Con relación a los impactos, se trabaja desde encuestas de percepción que permiten saber si las comunidades han sin atendidas y se encuentran satisfechas, evidenciando que se trabaja desde necesidades puntuales, siendo este punto la principal fragilidad del sistema. Los proyectos deberían alinearse a las políticas públicas del Estado o a los lineamientos generales de RSU propuestos por cada Universidad. Otra fragilidad importante es que las universidades del país estaban siendo evaluadas, lo que priorizó a los sistemas de evidencias y documentos, dejando de lado procesos de mejora o de reflexión sobre las acciones realizadas; además, generando miedo en la difusión de datos, dificultando la obtención de información, y al fortalecimiento de la RSU. Con autodiagnósticos fuertes se puede priorizar los planes de mejora, legitimar a la Universidad, siendo capaces de evaluar los impactos sociales y ambientales que se realizan (coherencia).

En el marco propuesto las Universidades ya no pueden solo transferir conocimiento, sino deben fomentar la relación con la sociedad, para fortalecer las estructuras sociales (Ramallo , 2015); Está claro que, sobre todo en contextos desiguales que la RSU puede contribuir a generar ciudades más justas con un desarrollo más equilibrado de la sociedad, de brindar un carácter participativo y contextualizado al entorno que les rodea y comprometer a todos los actores sobre los impactos que se producen, en especial aquellos relacionadas con temas de salud, bienestar y ciudadanía, involucrando una mirada desde el otro en la gestión con personas.

Entre los retos pendientes, el componente ambiental juega un papel fundamental que hoy en día debe ser considerado a la hora de concienciar a la población. Una vía posible es la creación de cursos abiertos al público, realizados desde una óptica inter y transdisciplinar, con la doble finalidad de ofrecer ayuda técnica en temas de mejoramientos de vivienda y de espacio público, y de capacitar en temas de ciudadanía a la población; y por otro lado, sensibilizar a la población en términos de justicia socio - ambiental, donde las personas se vean como agentes de cambio 
en una ecología integral y de corresponsabilidad con el entorno. Hasta ahora se ha hablado desde una mirada externa de la RSU, pero también debe ser interna; es decir, evaluar de qué forma se incentiva a los profesores en temas de docencia, investigación e vinculación en relación con necesidades sociales, además, de promover un campus sostenible; la RSU implica hacerse cargo de la realidad en todos los sentidos.

Desde el lado del ApS, se puede observar que es un elemento que pretende articular una materia o proyecto (práctica) al servicio y aprendizaje. Como conocen, la esencia del ApS está en conectar el currículo con las necesidades reales de la sociedad, además, de contribuir en el desarrollo de las habilidades blandas. Esta pedagogía motiva al diálogo, al consenso, al escuchar, a la asertividad, a la empatía, al respeto, al compromiso, entre otras, todas ellas para fortalecer el trabajo colaborativo (Francisco Amat \& Moliner Miravet, 2019). Todas estas características se fortalecerán si se encuentran enmarcadas dentro de la RSU y la justicia socio - ambiental. Por tal razón la RSU no puede ser considerada solo como un elemento de extensión, sino como un articulador que permite fortalecer la coherencia universitaria. Para que la RSU funcione necesita articularse con cuatro compontes: la Universidad, el Estado, las Organizaciones y la Sociedad, cada uno aportando para la transformación de la realidad, no solo desde una mirada academicista y cuantitativa, sino de un verdadero aporte a la sociedad.

Es importante que la arquitectura recupere su vocación social, y no se esconda en la idea de genio creador que la aísla a los habitantes de los contextos, hay que repensar el trabajo del arquitecto desde la academia. La arquitectura se relaciona con procesos, con productos, con comportamientos, que tiene una incidencia y repercusión muy grande en la sociedad. La Universidad puede actuar como un agente de cambio para el futuro, ya que puede permitir el desarrollo comunitario y generar redes de confianza entre los ciudadanos.

Cabe recalcar que a pesar de cada institución tenga su enfoque, ya sea nacional, comercial, administrativo o humanista, la RSU marca un fuerte aspecto Social, sin embargo, debe estar acompañada con lo Ambiental, planteando un nuevo enfoque de Responsabilidad Socio Ambiental Universitaria (RSAU). La RSAU puede ser un agente de activación, y, de transformación en los próximos años, siendo necesaria su implementación y debate para fortalecerla, revalorando temas de identidad y de interculturalidad de los pueblos, e implementarlos en cada carrera para retroalimentar a los procesos universitarios. La RSAU aplicada en todas las disciplinas permitirá comprobar la coherencia institucional y su grado de compromiso con el medio exterior.

\section{Bibliografía}

BELTRÁN-LLEVADOR, J., IÑIGO-BAJO, E., y MATA-SEGREDA, A. (2014). "La responsabilidad social universitaria, el reto de cosntrucción permanente" en Revista Iberoamericana de Educación Superior UNAM, Vol. 5 Num. 14, p. 3-18.

CES. (2019). Reglamento de Régimen Académico . RPC-SO-08-No.111-2019 . Ecuador: Consejo de Educación Superior.

DE LA CUESTA, M. (2011). "Responsabilidad Social Universitaria" en Boletín Trimestral de la Fundación Carolina, p. 2-3.

Dónde estudiar arquitectura en Distrito Metropolitano de Quito. <https://www.cursosycarreras.com.ec/arquitectura-distrito-metropolitano-de-quito-CZ-24-8006> [Consulta: 3 de noviembre de 2019] 
EL COMERCIO. El 60\% de las edificaciones son informales en el Distrito Metropolitano de Quito. $<$ https://www.elcomercio.com/actualidad/municipio-quito-regulacion-viviendas-informales.html> [Consulta: 9 de octubre de 2019]

FRANCISCO AMAT, A., y MOLINER MIRAVET, L. (2019). "El aprendizaje en servicio en la Universidad: una estrategia en la formación de ciudadanía crítica" en Revista Electrónica Interuniversitaria de Formación del Profesorado, 13 (4). <https://www.redalyc.org/comocitar.oa?id=217015570006> [Consulta: 12 de diciembre de 2019]

GASCA - PLIEGO , E., y OLVERA - GARCÍA, J. (2011). "Construir ciudadanía desde las universidades, responsabilidad social universitaria y desafíos ante el siglo XXI"en Convergencia Revista de Ciencias Sociales, num. 56. Universidad Autónoma del Estado de México,p. 37 - 58.

GONZÁLEZ, J. C., YÉPEZ , V., y GARCÍA, E. (2019). "Vinculación con la colectividad: Una propuesta de Gestión" en Vinculación con la colectividad: Una propuesta de Gestión. Quito: Centro de Publicaciones PUCE. P. 7 - 57.

HIDALGO, C. "Responsabilidad Social Universitaria” en Ríos - Mantilla, R. [Entrevista: 4 de diciembre de 2019]

LARRÁN, M., y ANDRADES PEÑA, F. (2015). "Análisis de la responsaqbilidad social universitaria desde diferentes enfoques teóricos" en Universia No. 15, Vol. 6, p. 91-107.

LOES. (2010). Ley Orgánica de Educación Superior. Ecuador: Consejo de Educación Superior.

LÓPEZ, J. "Resposnabilidad Social Universaitaria" en Ríos - Mantilla, R. [Entrevista: 12 de diciembre de 2019]

MALDONADO , D., y ROJAS, A. "Responsabilidad Social Universitaria" en Ríos - Mantilla, R. [Entrevista: 10 de diciembre de 2019]

MARINEZ DE CARRASQUERO, C., MAVÁREZ, R., y OTROS. (2008). La responsabildiad social universitaria como estrategia de vincualción con el entorno social . FRONESIS, Revista de Filosofía Jurídica, Social y Política, Vol. 15, No.3, 81-103.

MARTÍNEZ, C., CARAVALLO , B., y CARRASQUERO, C. (2014). "Perspectivas de responsabilidad social universitaria y acción comunal” en Fronesis Vol. 21, Issue 2, p. 263-271.

MIÑO, A. "Resposnabilidad Social Universitaria" en Ríos - Mantilla, R. [Entrevista: 15 de enero de 2020]

PUCE. Pontificia Universidad Católica del Ecuador . <https://www.puce.edu.ec/> [Consulta: 10 de enero de 2020]

RAMALLO, M. (2015). "La evaluación de la Responsabilidad Social Universitaria”en Debate Universitario, Universidad de Belgrano, p. 25-38.

RÍOS MANTILLA, R., GONZÁLEZ, J., ARMIJOS , E., y OTROS. (2016). "Estrategias para el arquitecto interprte: El consultorio en el Laboratorio de los Paisajes Vivos" en Arquitecturas del Sur, vol. 34 (49), p. 2231.

ROSER HERNÁNDEZ, I. (2010). Responsabilidad Social Universitaria . La Coruña: Netbiblo.

RSU AUSJAL . Compendio de Experiencias Significativas de Responsabilidad Social Universitaria AUSJAL Edición 19. <http://compendiorsu.ausjal.net/presentacion/> [Consulta: 18 de noviembre de 2019]

SASIA, P., DE LA CRUZ, C., y VALLAEYS, F. (2009). Resposabilidad Social Universitaria. Manual de primeros pasos. México, D.F.: McGraw - Hill.

UCE. Universidad Central del Ecuador. <https://www.uce.edu.ec/> [Consulta: 6 de enero de 2020]

UDLA. Universiad de las Américas. <https://www.udla.edu.ec/> [Consulta: 8 de enero de 2020] 
UNESCO (1998). Conferencia Mundial sobre la Educación Superior. <http://www.unesco.org/education/educprog/wche/principal/autonm-s.html> [Consulta: 20 de febrero de 2020]

USFQ. Universidad San Francisco de Quito. <http://www.usfq.edu.ec/Paginas/Inicio.aspx> [Consulta: 8 de enero de 2020] 